OPEN ACCESS

Edited by:

Marco Colizzi,

University of Verona, Italy

Reviewed by:

Anna Brancato,

University of Palermo, Italy

Anushree N. Karkhanis,

Binghamton University, United States

*Correspondence:

Nora D. Volkow

nvolkow@nida.nih.gov

Peter Manza

peter.manza@nih.gov

tThese authors have contributed equally to this work

Specialty section:

This article was submitted to

Addictive Disorders,

a section of the journal

Frontiers in Psychiatry

Received: 17 December 2020

Accepted: 13 April 2021

Published: 13 May 2021

Citation:

McPherson KL, Tomasi DG,

Wang G-J, Manza P and Volkow ND

(2021) Cannabis Affects Cerebellar

Volume and Sleep Differently in Men and Women.

Front. Psychiatry 12:643193

doi: 10.3389/fpsyt.2021.643193

\section{Cannabis Affects Cerebellar Volume and Sleep Differently in Men and Women}

\author{
Katherine L. McPherson ${ }^{1}$, Dardo G. Tomasi ${ }^{1}$, Gene-Jack Wang ${ }^{1}$, Peter Manza ${ }^{1 * t}$ and \\ Nora D. Volkow ${ }^{1,2 * t}$
}

${ }^{1}$ National Institute on Alcohol Abuse and Alcoholism, National Institutes of Health, Bethesda, MD, United States, ${ }^{2}$ National Institute on Drug Abuse, National Institutes of Health, Bethesda, MD, United States

Background: There are known sex differences in behavioral and clinical outcomes associated with drugs of abuse, including cannabis. However, little is known about how chronic cannabis use and sex interact to affect brain structure, particularly in regions with high cannabinoid receptor expression, such as the cerebellum, amygdala, and hippocampus. Based on behavioral data suggesting that females may be particularly vulnerable to the effects of chronic cannabis use, we hypothesized lower volumes in these regions in female cannabis users. We also hypothesized poorer sleep quality among female cannabis users, given recent findings highlighting the importance of sleep for many outcomes related to cannabis use disorder.

Methods: Using data from the Human Connectome Project, we examined 170 chronic cannabis users (>100 lifetime uses and/or a lifetime diagnosis of cannabis dependence) and 170 controls that we attempted to match on age, sex, BMI, race, tobacco use, and alcohol use. We performed group-by-sex ANOVAs, testing for an interaction in subcortical volumes, and in self-reported sleep quality (Pittsburgh Sleep Questionnaire Inventory).

Results: After controlling for total intracranial volume and past/current tobacco usage, we found that cannabis users relative to controls had smaller cerebellum volume and poorer sleep quality, and these effects were driven by the female cannabis users (i.e., a group-by-sex interaction). Among cannabis users, there was an age of first use-by-sex interaction in sleep quality, such that females with earlier age of first cannabis use tended to have more self-reported sleep issues, whereas this trend was not present among male cannabis users. The amygdala volume was smaller in cannabis users than in non-users but the group by sex interaction was not significant.

Conclusions: These data corroborate prior findings that females may be more sensitive to the neural and behavioral effects of chronic cannabis use than males. Further work is needed to determine if reduced cerebellar and amygdala volumes contribute to sleep impairments in cannabis users.

Keywords: marijuana, tetrahydrocannabinol, magnetic resonance imaging, sexual dimorphism, subcortical volume 


\section{INTRODUCTION}

There are marked sex differences in the acute and long-term effects of drugs of abuse, including subjective effects, neurological impact, and behavioral outcomes. These disparate effects may be due to differences in metabolism, body fat and water distribution, hormones, and sexual dimorphism in brain function. For example, differences in metabolism and bioavailability cause higher blood alcohol levels in females and greater vulnerability to the negative effects of alcohol, compared to males consuming the same amount of alcohol $(1,2)$. Greater drug effects in females are thought to contribute to "telescoping," the observation that women tend to progress from first use to seeking treatment for cannabis use disorder (CUD) more rapidly than men. This phenomenon has been described across several drugs of abuse, including cannabis use disorders (CUD) (3). Despite this, the prevalence of cannabis use and CUD is higher in males than females (4), which is driven by a greater rate of drug initiation among men than women, though this gap is narrowing (5). Along with this acceleration to CUD, women also experience stronger cannabis withdrawal symptoms than men during periods of abstinence (5), as well as worse outcomes on experimental cannabis therapies such as buspirone (6) and vilazodone (7).

These differences in responses to cannabis are likely related in part to sex differences in the function and structure of subcortical brain regions rich in cannabinoid-type I receptors (CB1-R, the primary receptor target for THC, the main psychoactive component of cannabis), such as the cerebellum, amygdala, and hippocampus (8). For instance, rats repeatedly treated with THC exhibited CB1-R desensitization and downregulation in cerebellum, hippocampus, prefrontal cortex, and striatum, with greater effects in females consistent with the "telescoping" observation (9) and which may be dependent on the estrous cycle (10). Chronic THC treatment also had lasting effects in primates, with THC concentration in the cerebellum approximately double the concentration in blood $24 \mathrm{~h}$ after the last dose of THC, indicating that brain regions with high CB1R density can be impacted long after cannabis use (11). Importantly, in individuals with CUD the cerebellum showed significant reductions in brain glucose metabolism during withdrawal whereas its activation during cannabis intoxication was associated with its reinforcing effects (12). Moreover, it has been proposed that the effects of cannabis on the cerebellum are relevant to cannabis addiction (13). As it relates to sex differences brain imaging studies showed that in individuals with CUD, females compared to males showed a blunted metabolic response to a stimulant challenge, which was most prominent in CB1-R-dense regions: cerebellum, hippocampus, and thalamus (14). Sex differences in the brain and behavior of cannabis users may also be critically related to sex differences in sleep quality, which is recognized as a factor impacting long-term outcomes in people with CUD (15). However, very little work has been done to describe the possible neurobiological underpinnings of sex differences in humans with a history of chronic cannabis use.

A broad literature has been devoted to understanding the effects of cannabis use on subcortical brain volumes. Findings have been inconsistent, with some studies finding substantially smaller subcortical volumes in chronic cannabis users compared to controls (16-18), whereas others have reported that after controlling for key confounding variables like tobacco usage, these differences are virtually non-existent $(19,20)$. We and others have argued that these discrepant findings are due to generally small sample sizes and inadequate matching on control groups (21). Nevertheless, several recent reviews have been devoted to the topic (22-24) and some consensus seems to have emerged that cerebellum, amygdala, and hippocampus volumes appear to be most consistently affected by chronic cannabis use across studies (8). However, whether these differences are moderated by sex, and are associated with behavioral outcomes such as sleep quality remains unknown.

Current findings regarding cannabis use and sleep quality are mixed, particularly when considering sex differences. Previous studies using the Pittsburgh Sleep Quality Index self-report scale (PSQI) among generally healthy adults, reported that women had lower scores on sleep quality (25-28), sleep efficiency (27), and higher sleep disturbances (28) than men, suggesting that sex differences in sleep quality may exist even before taking substance use into account. Chronic cannabis use can further complicate this picture. Acute withdrawal from cannabis can contribute to objective and subjective sleep disturbances, which are more common in chronic users $(29,30)$. Acutely cannabis can decrease sleep latency, making it easier to fall asleep $(31,32)$; however, long-term sleep quality is negatively impacted (15). In fact, roughly half of adults with CUD reported that cannabis use had caused them difficulty sleeping in the past 90 days (33). Heavy users also reported a decrease in desirable sleep aftereffects (e.g., restful sleep, duration) over time (34). Females compared to males who had "risky" use of both alcohol and cannabis reported especially poor sleep quality reflected by high PSQI total scores (35), but it was not clear whether alcohol or cannabis use was most associated with this pattern. In sum, while cannabis use and sex can have strong effects on sleep quality, we are not aware of any studies that have investigated the interaction between these two factors. This is particularly relevant given a wide body of work that chronic impaired sleep quality can negatively impact brain structure [e.g., (36)].

Together, converging evidence suggests that there are sex differences in the effects of chronic cannabis use on subcortical brain volumes and sleep. However, the interaction of sex on cannabis effects on subcortical brain volumes and sleep quality has not been investigated. To address this neglect, we took advantage of Human Connectome Project data (37) to examine brain structure and sleep quality in a relatively large number of participants with a history of chronic cannabis use and wellmatched controls. We hypothesized that female cannabis users would have smaller volumes in amygdala, hippocampus, and cerebellum, which are subcortical regions dense with CB1-Rs (38), and poorer sleep quality than male cannabis users.

\section{MATERIALS AND METHODS}

\section{Participants}

Participants included in this study provided written informed consent at Washington University in St. Louis (39). Out of 1,005 
TABLE 1 | Demographics and clinical characteristics for chronic cannabis users (CAN) and controls (CTL).

\begin{tabular}{|c|c|c|c|c|}
\hline & Mean (SD) & Mean (SD) & M vs. F: T-stat, $p$ & CAN vs. CTL: T-stat, $p$ \\
\hline Cannabis (CAN) & Males $(n=114)$ & Females $(n=56)$ & & \\
\hline Age & 27.614 (3.635) & $28.714(3.944)$ & $-1.754,0.082$ & $-0.247,0.805$ \\
\hline BMl & $26.033(4.110)$ & 27.477 (6.320) & $-1.556,0.124$ & $-0.284,0.777$ \\
\hline Edu & 14.465 (1.825) & 14.018 (1.995) & $1.411,0.161$ & $-2.039,0.042$ \\
\hline Tobacco use (Composite-Z) & $0.647(1.082)$ & $0.366(1.096)$ & $1.578,0.118$ & $3.246,0.001$ \\
\hline Alcohol use (Composite-Z) & $0.218(0.424)$ & $0.061(0.341)$ & $2.600,0.010$ & $0.901,0.368$ \\
\hline \% Caucasian & 72.81 & 57.14 & $\chi^{2}=4.210, p=0.040$ & \\
\hline \% Black/African American & 15.79 & 30.36 & $\chi^{2}=4.874, p=0.027$ & \\
\hline Controls (CTL) & Males $(n=114)$ & Females $(n=56)$ & & \\
\hline Age & 27.658 (3.604) & $28.929(3.756)$ & $-2.101,0.038$ & \\
\hline BMl & 26.406 (3.953) & $27.163(5.048)$ & $-0.976,0.332$ & \\
\hline Edu & 14.702 (1.755) & 14.768 (1.849) & $-0.223,0.824$ & \\
\hline Tobacco use (Composite-Z) & $0.189(0.987)$ & $0.191(0.976)$ & $-0.015,0.988$ & \\
\hline Alcohol use (Composite-Z) & $0.184(0.376)$ & $0.018(0.249)$ & $3.431,0.001$ & \\
\hline$\%$ Caucasian & 74.56 & 58.93 & $\chi^{2}=4.323, p=0.038$ & \\
\hline \% Black/African American & 15.79 & 30.36 & $\chi^{2}=4.874, p=0.027$ & \\
\hline
\end{tabular}

individuals with structural MRI data in the Human Connectome Project, we identified 170 individuals meeting DSM-IV criteria for lifetime (current or prior) CUD and/or $>100$ lifetime cannabis uses, and without comorbid current or prior alcohol dependence, as in our previous work $(21,40)$, which became the cannabis group (CAN). We also selected a control group (CTL; $n=170$ ) with $<10$ lifetime cannabis uses, and used the matchControls package in $\mathrm{R}$ to try and match controls with the CAN group on: age, sex, education, BMI, race, and a composite measure reflecting past/current alcohol usage $(41,42)$. Of note, we could not match on tobacco usage, which was higher in the CAN group $(p<0.001)$, and subsequent analyses were performed to ensure results were not driven by past/current tobacco usage. For more details on participant demographics see Table 1.

\section{MRI Image Acquisition and Preprocessing}

Scans were collected using a custom-made Siemens Connectom Skyra scanner with a 32-channel head coil. T1- and T2-weighted anatomical scans were acquired at $0.7 \mathrm{~mm}$ isotropic resolution (37). Structural images were "minimally preprocessed" by HCP investigators through standardized pipelines (43). Images were corrected for gradient non-linearity-induced distortions, readout distortions, and intensity inhomogeneities, and then aligned to the MNI atlas. Then, images were processed through a customized version of Freesurfer. We used the volume values for all subcortical regions (averaged across the left and right regions, where possible) in the Desikan-Killany parcellation (44), which resulted in the analysis of 10 regions: Amygdala, Hippocampus, Putamen, Caudate, Nucleus Accumbens, Thalamus, Pallidum, Brainstem, Cerebellar Cortex, and Cerebellar White Matter.

\section{Self-Reported Sleep Quality}

The Pittsburgh Sleep Quality Index (PSQI) was developed in 1988 to assess sleep via 19 questions; it produces a validated global score based on seven sub-scores such as efficiency, quality, and disturbances (45).

\section{Statistical Analyses}

Analyses were performed in R version 3.6.2 and in GraphPad Prism version 8.0.1. To test for sex differences in subcortical regional volumes, we constructed linear regression models using the $\operatorname{lm}$ Function in $\mathrm{R}$ (equivalent to an analysis of variance), where the main effects of sex and cannabis group membership (and their interaction) were the predictor variables, tobacco usage and total intracranial volume were covariates, and each region's subcortical volume was the outcome variable. To correct for multiple comparisons across all 10 regions of interest, we used false discovery rate (Benjamini-Hochberg) correction. We also tested for differences in self-reported sleep quality using the same analytical approach, except that total PSQI score was the outcome variable.

To attempt to link any of the above findings that showed significant cannabis group-by-sex interactions, we performed mediation analysis. We tested whether sleep scores mediated the association between sex and subcortical volumes, using the causal mediation analysis toolbox in R (46) with 1,000 permutations. We also tested the reverse mediation analysis: that subcortical volumes mediated the association between sex and self-reported sleep quality. In these analyses we used only the data from participants in the CAN group $(n=170)$, and we controlled for tobacco usage and total intracranial volume.

Finally, we tested if any of the subcortical volumes or selfreported sleep quality with significant cannabis group-by-sex interactions were driven by participants who had an earlier age of cannabis use onset, since this has been associated with poorer outcomes in cannabis users generally, and in our prior study with differences in subcortical function (42). The HCP recorded age 


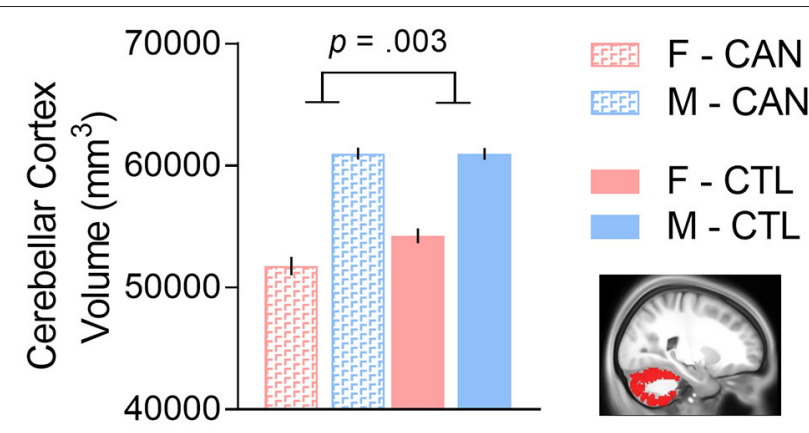

FIGURE 1 | There was a cannabis group-by-sex interaction in cerebellar cortex volume, such that females with a history of chronic cannabis use had smaller volumes than the other groups. We controlled for tobacco usage and total intracranial volume in the analysis. F, Female; M, Male; CAN, Chronic cannabis use group; CTL, control group.

of first cannabis use on an ordinal scale (1: <14 years old, $2: 15-$ 17 years old, 3: 18-20 years old, $4: 21+$ years old). We therefore tested for interaction effects by performing sex-by-age of first use ANOVAs, using only the data from participants in the cannabis use group $(n=170)$, again controlling for tobacco usage and total intracranial volume.

\section{RESULTS}

We constructed linear models to determine if the interaction of sex and chronic cannabis usage was significantly associated with subcortical volumes, controlling for tobacco usage and total intracranial volume. We first noted that there were no significant main effects of sex in any of the 10 regions tested after FDR correction (all $p$ 's $>0.20$ ). There was a main effect of group in the cerebellar cortex $\left[t_{(1,334)}=-3.353\right.$, FDR-corrected $p=$ 0.008 ], which was driven by the female cannabis users having lower cerebellar volumes than the other participants [interaction effect: $t_{(1,334)}=-3.699$, FDR-corrected $p=0.002$; Figure 1]. There was also a trend for a main effect of group in the amygdala $\left[t_{(1,334)}=-2.611\right.$, FDR-corrected $\left.p=0.047\right]$, with CAN having lower amygdala volumes than controls, but the sex interaction effect was not significant. No other region (including amygdala) showed a significant group or interaction effect (all $p$ 's $>0.35$; for full results, see Table 2 ).

We further tested whether the interaction of sex and chronic cannabis usage was associated with self-reported sleep quality, again controlling for tobacco usage and total intracranial volume. There was no significant main effect of sex $\left[t_{(1,334)}=1.323\right.$, $p=0.187]$, however there was a main group effect $\left[t_{(1,334)}=\right.$ $3.233, p=0.001$ ], which was also driven by the female cannabis users having poorer sleep quality than the other participants [interaction effect: $t_{(1,334)}=-2.208, p=0.028$; Figure 2]. In exploratory analysis, we tested whether cerebellum volume was correlated with self-reported sleep quality among the female cannabis users only, but did not observe a significant effect: $t_{1,52}=0.418, p=0.677$.
TABLE 2 | Summary statistics for analysis of subcortical regional volumes, showing: (1) the main effects of group, i.e., the chronic cannabis use group (CAN) vs. controls (CTL); (2) the main effect of sex, i.e., Males (M) vs. Females (F); and (3) their interaction.

\begin{tabular}{|c|c|c|c|}
\hline Region & $\begin{array}{l}\text { Group: CAN } \\
>\text { CTL } t\left(p_{a d j}\right)\end{array}$ & $\begin{array}{l}\text { Sex: } M>F \\
t\left(p_{a d j}\right)\end{array}$ & $\begin{array}{l}\text { Interaction: } t \\
\left(p_{a d j}\right)\end{array}$ \\
\hline Cerebellar cortex & $-3.353(0.009)$ & $1.82(0.232)$ & $3.699(0.003)$ \\
\hline Cerebellar WM & -0.835 (0.763) & $1.126(0.473)$ & $1.323(0.374)$ \\
\hline Amygdala & $-2.611(0.047)$ & $1.884(0.232)$ & $1.78(0.374)$ \\
\hline Hippocampus & $-1.341(0.603)$ & $1.228(0.473)$ & $1.434(0.374)$ \\
\hline Putamen & $0.623(0.763)$ & $1.85(0.232)$ & $-0.1(0.921)$ \\
\hline Caudate & -0.422 (0.842) & $-0.974(0.473)$ & $0.413(0.756)$ \\
\hline Accumbens & -1.101 (0.679) & $1.059(0.473)$ & $1.446(0.374)$ \\
\hline Thalamus & $-0.266(0.871)$ & $0.379(0.881)$ & $0.681(0.633)$ \\
\hline Pallidum & $0.731(0.763)$ & -0.07 (0.998) & $0.665(0.633)$ \\
\hline Brainstem & $0.162(0.871)$ & $-0.003(0.998)$ & $0.911(0.605)$ \\
\hline
\end{tabular}

All $p$-values are adjusted using False Discovery Rate Benjamini-Hochberg Correction. WM, White Matter. Bold values denote statistical significance $(p<0.05)$.

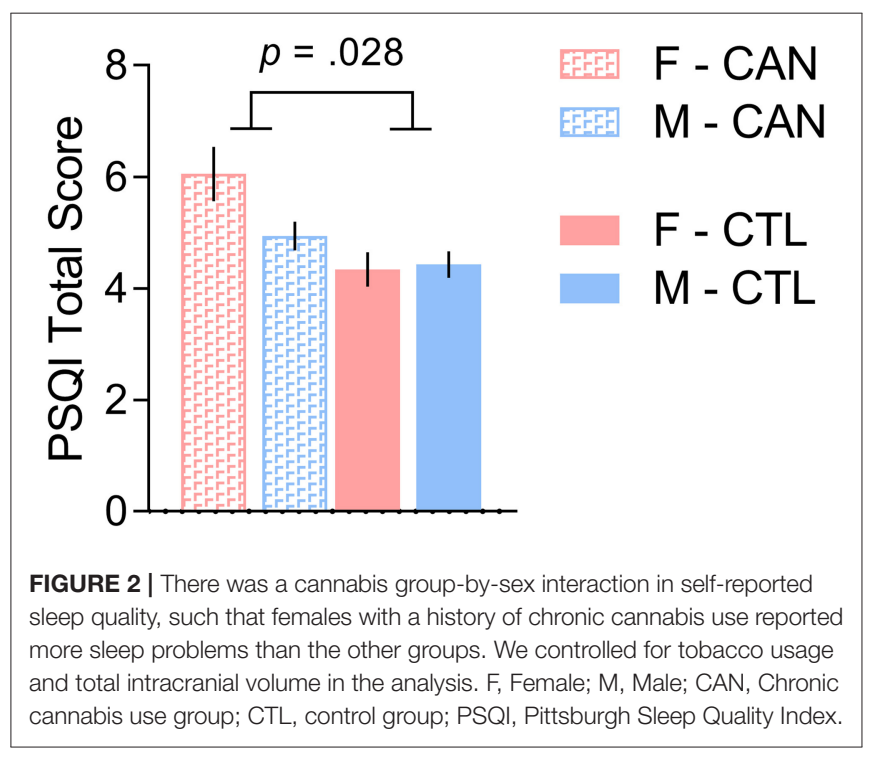

However, there were no significant mediation effects in the models we tested. Among the cannabis group only $(n=170)$, sleep scores (total PSQI score) did not significantly mediate the sex differences in cerebellar volume: [mediation effect estimate $=15.20,95 \% \mathrm{CI}=(-208.0,202.6), p=0.93$; direct effect estimate $=4,950,95 \% \mathrm{CI}=(3,030,6,890), p<1 \times$ $\left.10^{-16}\right]$. Likewise, in the reverse model, cerebellar volumes did not significantly mediate the sex differences in sleep scores: [mediation effect estimate $=-0.064,95 \% \mathrm{CI}=(-0.607,0.470)$, $p=0.82$; direct effect estimate $=-0.510,95 \% \mathrm{CI}=(-2.045$, $0.980), p=0.58]$.

Finally, we tested if the significant interaction results in cerebellum volume and sleep were driven by female participants who had an earlier age of cannabis use onset in the CAN group $(n=170)$. The cerebellar volumes showed significant main effects of sex $\left[F_{(1,160)}=168.764, p<1 \times 10^{-16}\right]$ and age of first cannabis 


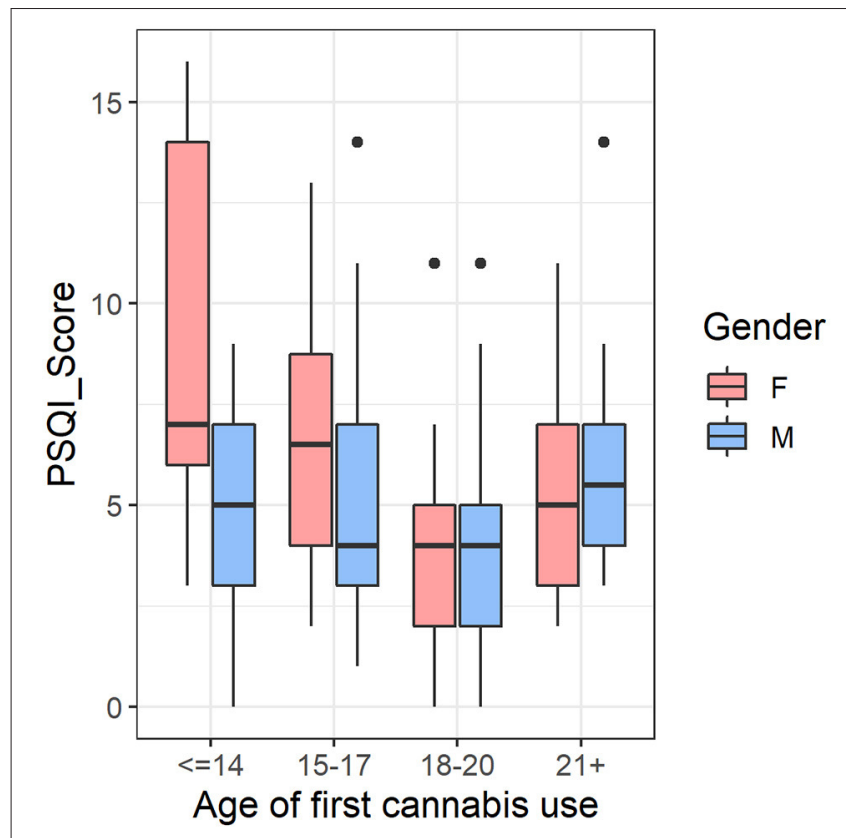

FIGURE 3 | In the cannabis group (CAN) $(n=170)$, there was an age of first use-by-sex interaction in self-reported sleep quality, such that females with earlier age of first cannabis use tended to have more self-reported sleep issues, whereas this trend was not present in male cannabis users. We controlled for tobacco usage and total intracranial volume in the analysis. F, Female; M, Male; PSQI, Pittsburgh Sleep Quality Index.

use $\left[F_{(3,160)}=3.812, p=0.011\right]$ but their interaction was not significant $\left[F_{(3,160)}=1.583, p=0.196\right]$. However, for total PSQI score, we observed significant main effects of sex $\left[F_{(1,160)}=5.179\right.$, $p=0.024]$, age of first use $\left[F_{(3,160)}=4.077, p=0.008\right]$, and their interaction, $\left[F_{(3,160)}=3.587, p=0.015\right]$, such that females with earlier age of first cannabis use tended to have more self-reported sleep issues, whereas this trend was not present in male cannabis users (Figure 3).

\section{DISCUSSION}

Our investigation of the impact of cannabis abuse on various subcortical regions yielded results that add to a body of recent work using Human Connectome Project (HCP) data. For instance, recent cannabis use in this sample was negatively associated with hippocampal volume (47) and smaller left hippocampal volume mediated the association between frequency of cannabis use and working memory deficits in cannabis users (48). Additionally, HCP data has revealed an effect of THC exposure on amygdala microstructure organization (49). In line with these studies, we found that cannabis users had marginally smaller amygdala volumes than non-users. However, we only found a cannabis use-by-sex interaction in cerebellar cortex volumes, suggesting that females may be particularly susceptible to the effects of chronic cannabis use in this region. Finally, we observed that female cannabis users had poorer self-reported sleep quality than the other groups, which was particularly pronounced among females who began using cannabis in early adolescence. We discuss these findings in more detail below.

The cerebellum has traditionally been studied for its role in balance and motor coordination (50), nociception (51), and motor cognition $(52,53)$. Brain imaging studies in humans have shown that the cerebellum is sensitive to the acute and chronic effects of cannabis (8), including glucose metabolic activity (12, $54)$, volume, and resting-state activity (13, 55-57). Postmortem studies have found striking differences in the cerebellar structure of drug abusers relative to controls; one group showed increased autophagy biomarkers in the cerebellum of multi-substance drug abusers (58), while another found signs of neurodegeneration in the cerebellar cortex of people who were dependent on opioids, suggesting that drug addiction can negatively impact cerebellar structure (59). Recently, Gil-Miravet et al. found that the cerebellum modulates drug-cue associative memory in cocaine users (60), while Hung et al. showed increased functional connectivity between the pallidum and cerebellum of ketamine users, suggesting that the cerebellum has a fundamental role in the pathophysiology of addiction (61). The cerebellum is clearly affected by cannabis use as well; chronic cannabis users can experience cerebellar-dependent motor adaptation impairment (62), while synthetic cannabinoid users show reduced gray matter volume in the left cerebellum (63). These studies are consistent with several recent reviews published on the topic which note the cerebellum's role as a nexus between motor, reward, and cognitive processes crucial to drug seeking behavior (64-66). Compared to other brain regions, there is a relatively high concentration of CB1-Rs in the cerebellum $(38,67,68)$. PET studies have shown that $\mathrm{CB}-1 \mathrm{Rs}$ are reversibly downregulated in people with a history of chronic cannabis consumption, which is likely to contribute to tolerance and dependence with repeated use (69). Previous studies have had mixed findings on the relationship between chronic cannabis use and cerebellar volumes with some studies suggesting that cannabis actually increases gray matter volumes $(57,70-73)$. Here we found that the smaller cerebellar cortical volumes in cannabis users relative to the controls were driven by the female cannabis users. This could explain the discrepancies in the literature since sex was not accounted for in prior investigations. Indeed, studies finding larger cerebellar volumes in cannabis users had very few or no female participants in the cannabis group: Wang et al.: $25 \%$ female $(5 \mathrm{~F} / 15 \mathrm{M})$; Cousijn et al.: $36 \%$ Female (12F/21M); Battistella et al.: 0\% Female (0F/31M); Wu and Yang: $25 \%$ female $(5 \mathrm{~F} / 15 \mathrm{M})$; Koenders et al.: $25 \%$ female $(5 \mathrm{~F} / 15 \mathrm{M})$. Those findings contrast with the results in our $33 \%$ Female sample $(56 \mathrm{~F} / 114 \mathrm{M})$ and those of another related study (50\% Female; $13 \mathrm{~F} / 13 \mathrm{M})$ that found lower cerebellar microstructural integrity in adults at risk for CUD relative to controls (56). These findings underscore the importance of including an adequate number of female participants and of investigating sex differences in brain and behavioral outcomes for people with chronic substance use, for such differences appear to be prevalent throughout the addiction endophenotype $(8,74,75)$.

We also observed a trend for a group effect on amygdala volume, with lower volumes in cannabis users compared to healthy controls, in agreement with prior studies $(73,76)$, and 
which correlated with amount of cannabis used and dependence severity $(70,73)$. In terms of sex differences, one study found that while adolescent female cannabis users had larger right amygdalar volumes than healthy controls, there was no such difference in males (77). However, our finding of lower amygdalar volume in cannabis users was not sex-dependent and likely would not contribute to the sex-dependent impairment in sleep quality that we observed. Nonetheless, it is possible that a deficit in amygdala volume could contribute to the overall poorer sleep quality observed in CUD compared to controls. The amygdala has been previously implicated in poor sleep quality; while functional connectivity between the amygdala and premotor cortex is negatively associated with sleep quality (78), it appears that sleep quality might modulate amygdalar functional connectivity and not vice versa (79). Additionally, patients with narcolepsy have lower GM volume in the amygdala relative to controls, suggesting a possible unidirectional relationship between sleep quality and amygdala volume (80).

Our finding of sex-specific differences in cerebellar volume among cannabis users was not present in other regions with high CB1-R density, such as the amygdala and the hippocampus. Preclinical studies in rats have shown that chronic THC caused downregulation and desensitization of CB1-R in cerebellum, and these decreases were especially large in females (9). Other studies have reported higher baseline CB1-R density in female compared to male rats, although the cerebellum was not examined (10). Human PET studies have similarly found that females have higher baseline CB1-R availability than males in many brain regions (81), including cerebellar cortex (82). Given that CB1$\mathrm{R}$ density was influenced by the estrous cycle in preclinical studies, it is possible that female sex hormones play a role in sex differences in CB1-R availability as well as sex differences on cannabis effects in brain and behavior (10). Animal models could be used to test if sex differences in CB1-R density prior to and after chronic THC exposures may confer female vulnerability to potential neurotoxic effects of cannabis on cerebellar structure and function. Additionally, while initial human PET studies found that CB1-Rs in both sexes were downregulated in response to chronic cannabis use (69), future longitudinal studies should examine whether there are sex differences on the association of CB1-R downregulation with the severity of CUD. This is especially important given that there are currently no FDAapproved pharmacological treatments for CUD, and that one promising candidate, the fatty-acid amide hydrolase (FAAH) inhibitor PF-04457845, was recently shown to reduce cannabis withdrawal severity and promote abstinence, but only men with CUD were included in the trial (83). Thus, much work remains to be done to see if treatments show similar improvements in females and if they do so in part via cerebellar mechanisms. Our group has previously proposed that downregulation of CB1R in subjects with cannabis dependence might increase vulnerability to cortical thinning, suggesting that CB1R availability can lead to structural changes in the brain (21). Another study found that some heavy cannabis users have a genetic predisposition toward cannabis dependence due to a functional single nucleotide polymorphism affecting cannabis receptor-1 gene expression; among cannabis users, minor relative to major allele carriers had lower volume in the nearby hippocampus, but not the amygdala (84). However, cerebellum volume was not examined in this study, and it is possible that there is a similar connection between CB1R and cerebellar volume. Future studies should examine this possibility to uncover the link between CB1R activation/availability and amygdalar/cerebellar volume.

We also observed that females' self-reported sleep quality (as indexed by the global PSQI score) was similarly more vulnerable to the negative effects of cannabis use than for males. Given that patients with degenerative diseases of the cerebellum such as cerebellar ataxia commonly report sleep disturbances, poor subjective sleep quality, restless leg syndrome, and REM behavior disorder, it is plausible that the cerebellar volume loss in female cannabis users contributed to their poor sleep quality $(85,86)$. However, in our study the effects of cannabis on cerebellar volumes did not mediate the effects of cannabis on sleep quality, which is likely to reflect a more complex association between cannabis effects in brain structure and function. Similarly, the effects of cannabis on sleep quality did not mediate its effects on brain volume, which might also indicate distinct neurobiological processes underlying these two effects. Given the observational nature of this study, we are unable to rule out the possibility that women to start with had lower sleep quality than men as has been reported by other studies (25-28), though in our current study sleep scores in control males did not significantly differ from those in control females. It is also possible that a mismatch between expectation and reality in how cannabis helps with sleep may play a role in self reports; in a majority female (67\%) sample of cannabis users, while both frequency and presence of cannabis use were associated with the expectation of improved sleep, cannabis use was actually associated with poorer subjective sleep quality (87). Finally, we observed that females who reported first using cannabis in early adolescence tended to report the worst sleep quality, which aligns with a recent large-scale twin study $(n=1,656)$ that reported that regular cannabis use at a young age correlated with shorter sleep duration in adulthood (88). Given the differences in socialization, development, and expectations associated with cannabis use, women may be more vulnerable to the negative sleep effects of cannabis abuse at younger ages than men. These data complement a large body of literature suggesting that early-onset cannabis use is strongly associated with poor neuropsychiatric outcomes (89), and again highlight sex differences as an important future avenue of investigation.

\section{Limitations}

The HCP provides a large, high-quality dataset of MRIbased and behavioral data (90). Nonetheless, given that scans were completed between 2012 and 2015 by the WUMinn Consortium, in Missouri and Minnesota, where medical marijuana was not legalized until 2014 (albeit restrictively and only for certain chronic conditions) it is likely that most participants used cannabis recreationally, not as prescribed by a doctor. While this allows us to compare a uniform population of chronic recreational users to non-users, we were unable to investigate any effects of medical cannabis use on sleep quality or cerebellar volumes. We also do not have any information on whether participants were using cannabis to self-treat sleep 
issues. It is possible that when used for medicinal purposes and with low-THC strains that are less likely to lead to CUD (91), cannabis may not have a negative impact on sleep (15). Additionally, while we matched the chronic cannabis use group with controls on several important demographic variables including a composite score reflecting current and past alcohol consumption, cannabis users did not match controls on measures of tobacco usage. Considering that females had lower nicotine use than males and yet they showed greater effects than males, and that we covaried for tobacco use, it is likely that the effects on sleep and cerebellar volumes reflect cannabis and not nicotine effects. Nonetheless we cannot completely rule out that the interaction between cannabis and tobacco use contributed to the effects in brain and sleep quality. Finally, this analysis is limited by the imbalance in the number of male and female subjects in our sample: each one of the groups had twice as many males as females. This sex imbalance is representative of the U.S. population at large, since the majority of people who use cannabis are male (92-98), though this imbalance may affect our results on group differences between cannabis users and controls (as noted in the discussion) and limit our statistical power. This limitation emphasizes the need to include equal numbers of men and women in clinical studies, so that sex differences can be rigorously examined.

\section{Future Directions}

Future studies should include polysomnography measurements or other objective measures of sleep architecture and duration in addition to self-reported sleep data. The impact of cannabis use on sleep requires further exploration, for a recent metaanalysis reported that most of the prior studies reported sleep as a secondary outcome and were done on small sample sizes using unvalidated measures (99). Further, studies on the effects of cannabis on sleep architecture and its response to treatment

\section{REFERENCES}

1. Baraona E, Abittan CS, Dohmen K, Moretti M, Pozzato G, Chayes ZW, et al. Gender differences in pharmacokinetics of alcohol. Alcohol Clin Exp Res. (2001) 25:502-7. doi: 10.1111/j.1530-0277.2001.tb02242.x

2. Frezza M, di Padova C, Pozzato G, Terpin M, Baraona E, Lieber CS. High blood alcohol levels in women. N Engl J Med. (1990) 322:95-9. doi: 10.1056/NEJM199001113220205

3. Hernandez-Avila CA, Rounsaville BJ, Kranzler HR. Opioid-, cannabisand alcohol-dependent women show more rapid progression to substance abuse treatment. Drug Alcohol Depend. (2004) 74:265-72. doi: 10.1016/j.drugalcdep.2004.02.001

4. Khan SS, Secades-Villa R, Okuda M, Wang S, Pérez-Fuentes G, Kerridge BT, et al. Gender differences in cannabis use disorders: results from the national epidemiologic survey of alcohol and related conditions. Drug Alcohol Depend. (2013) 130:101-8. doi: 10.1016/j.drugalcdep.2012. 10.015

5. Cooper ZD, Craft RM. Sex-dependent effects of cannabis and cannabinoids: a translational perspective. Neuropsychopharmacology. (2018) 43:34-51. doi: $10.1038 /$ npp. 2017.140

6. McRae-Clark AL, Baker NL, Gray KM, Killeen TK, Wagner AM, Brady KT, et al. Buspirone treatment of cannabis dependence: a randomized, placebo-controlled trial. Drug Alcohol Depend. (2015) 156:2937. doi: 10.1016/j.drugalcdep.2015.08.013 are sorely needed. Finally, future studies should attempt to account for THC potency and a richer quantification of doses and frequency of cannabis use (100), to discern the effects of light vs. heavy cannabis use in general and in the context of these sex-dependent effects on sleep and cerebellar volume.

\section{DATA AVAILABILITY STATEMENT}

The original contributions presented in the study are included in the article/supplementary material, further inquiries can be directed to the corresponding author/s.

\section{ETHICS STATEMENT}

The studies involving human participants were reviewed and approved by Washington University in St Louis IRB. The patients/participants provided their written informed consent to participate in this study.

\section{AUTHOR CONTRIBUTIONS}

KM, PM, G-JW, and NV: study conception. KM, PM, and DT: data analysis. KM: first draft of manuscript. All authors: editing.

\section{FUNDING}

Data were provided by the Human Connectome Project, WU-Minn Consortium (Principal Investigators: David Van Essen and Kamil Ugurbil; 1U54MH091657) funded by the 16 $\mathrm{NIH}$ Institutes and Centers that support the NIH Blueprint for Neuroscience Research; and by the McDonnell Center for Systems Neuroscience at Washington University. This work was supported by the National Institute on Alcohol Abuse and Alcoholism (Grant No. Y1AA-3009).
7. McRae-Clark AL, Baker NL, Gray KM, Killeen T, Hartwell KJ, Simonian SJ. Vilazodone for cannabis dependence: a randomized, controlled pilot trial. Am J Addict. (2016) 25:69-75. doi: 10.1111/ajad. 12324

8. Ferland JN, Hurd YL. Deconstructing the neurobiology of cannabis use disorder. Nat Neurosci. (2020) 23:600-10. doi: 10.1038/s41593-020-0611-0

9. Farquhar CE, Breivogel CS, Gamage TF, Gay EA, Thomas BF, Craft RM, et al. Sex, THC, and hormones: effects on density and sensitivity of CB1 cannabinoid receptors in rats. Drug Alcohol Depend. (2019) 194:20-7. doi: 10.1016/j.drugalcdep.2018.09.018

10. Castelli M, Fadda P, Casu A, Spano M, Casti A, Fratta W, et al. Male and female rats differ in brain cannabinoid CB1 receptor density and function and in behavioural traits predisposing to drug addiction: effect of ovarian hormones. Curr Pharm Des. (2014) 20:2100-13. doi: $10.2174 / 13816128113199990430$

11. Withey SL, Bergman J, Huestis MA, George SR, Madras BK. THC and CBD blood and brain concentrations following daily administration to adolescent primates. Drug Alcohol Depend. (2020) 213:108129. doi: 10.1016/j.drugalcdep.2020. 108129

12. Volkow ND, Gillespie H, Mullani N, Tancredi L, Grant C, Valentine A, et al. Brain glucose metabolism in chronic marijuana users at baseline and during marijuana intoxication. Psychiatry Res Neuroimaging. (1996) 67:29-38. doi: 10.1016/0925-4927(96)02817-X 
13. Moreno-Rius J. The cerebellum, THC, and cannabis addiction: findings from animal and human studies. Cerebellum. (2019) 18:593-604. doi: 10.1007/s12311-018-0993-7

14. Wiers CE, Shokri-Kojori E, Wong CT, Abi-Dargham A, Demiral SB, Tomasi D, et al. Cannabis abusers show hypofrontality and blunted brain responses to a stimulant challenge in females but not in males. Neuropsychopharmacology. (2016) 41:2596-605. doi: 10.1038/npp.2016.67

15. Babson KA, Sottile J, Morabito D. Cannabis, cannabinoids, and sleep: a review of the literature. Curr Psychiatry Rep. (2017) 19:23. doi: 10.1007/s11920-017-0775-9

16. Yücel M, Lorenzetti V, Suo C, Zalesky A, Fornito A, Takagi MJ, et al. Hippocampal harms, protection and recovery following regular cannabis use. Transl Psychiatry. (2016) 6:e710. doi: 10.1038/tp.2015.201

17. Chye YY, Lorenzetti V, Suo C, Batalla A, Cousijn J, Goudriaan $\mathrm{AE}$, et al. Alteration to hippocampal volume and shape confined to cannabis dependence: a multi-site study. Addict Biol. (2018) 24:822-34. doi: $10.1111 /$ adb. 12652

18. Gilman JM, Kuster JK, Lee S, Lee MJ, Kim BW, Makris N, et al. Cannabis use is quantitatively associated with nucleus accumbens and amygdala abnormalities in young adult recreational users. J Neurosci. (2014) 34:552938. doi: 10.1523/JNEUROSCI.4745-13.2014

19. Weiland BJ, Thayer RE, Depue BE, Sabbineni A, Bryan AD, Hutchison KE. Daily marijuana use is not associated with brain morphometric measures in adolescents or adults. J Neurosci. (2015) 35:1505-12. doi: 10.1523/JNEUROSCI.2946-14.2015

20. Scott JC, Rosen AFG, Moore TM, Roalf DR, Satterthwaite TD, Calkins $\mathrm{ME}$, et al. Cannabis use in youth is associated with limited alterations in brain structure. Neuropsychopharmacology. (2019) 44:1362-9. doi: 10.1038/s41386-019-0347-2

21. Manza P, Yuan K, Shokri-Kojori E, Tomasi D, Volkow ND. Brain structural changes in cannabis dependence: association with MAGL. Mol Psychiatry. (2020) 25:3256-66. doi: 10.1038/s41380-019-0577-z

22. Lorenzetti V, Chye Y, Silva P, Solowij N, Roberts CA. Does regular cannabis use affect neuroanatomy? An updated systematic review and meta-analysis of structural neuroimaging studies. Eur Arch Psychiatry Clin Neurosci. (2019) 269:59-71. doi: 10.1007/s00406-019-00979-1

23. Zehra A, Burns J, Liu CK, Manza P, Wiers CE, Volkow ND, et al. Cannabis addiction and the brain: a review. Focus. (2019) 17:169-82. doi: 10.1176/appi.focus.17204

24. Nader DA, Sanchez ZM. Effects of regular cannabis use on neurocognition, brain structure, and function: a systematic review of findings in adults. Am J Drug Alcohol Abuse. (2018) 44:4-18. doi: 10.1080/00952990.2017.1306746

25. Li L, Sheehan CM, Thompson MS. Measurement invariance and sleep quality differences between men and women in the Pittsburgh sleep quality index. $J$ Clin Sleep Med. (2019) 15:1769-76. doi: 10.5664/jcsm.8082

26. Chen X, Fang Y, Liu X, Zhao D, Feng X, Li P. Gender differences in latent classes of sleep quality in community-dwelling adults based on the Pittsburgh sleep quality index. Psychol Health Med. (2019) 24:901-10. doi: 10.1080/13548506.2019.1634825

27. Lin J-N. Gender differences in self-perceptions about aging and sleep among elderly Chinese residents in Taiwan. J Nurs Res. (2016) 24:347-56. doi: 10.1097/JNR.0000000000000167

28. Morris JL, Rohay J, Chasens ER. Sex differences in the psychometric properties of the Pittsburgh sleep quality index. J Womens Heal. (2018) 27:278-82. doi: 10.1089/jwh.2017.6447

29. Angarita GA, Emadi N, Hodges S, Morgan PT. Sleep abnormalities associated with alcohol, cannabis, cocaine, and opiate use: a comprehensive review. Addict Sci Clin Pract. (2016) 11:9. doi: 10.1186/s13722-016-0056-7

30. Copersino ML, Boyd SJ, Tashkin DP, Huestis MA, Heishman SJ, Dermand JC, et al. Cannabis withdrawal among non-treatment-seeking adult Cannabis users. Am J Addict. (2006) 15:8-14. doi: 10.1080/10550490500418997

31. Chait LD. Subjective and behavioral effects of marijuana the morning after smoking. Psychopharmacology. (1990) 100:328-33. doi: 10.1007/BF02244601

32. Nicholson AN, Turner C, Stone BM, Robson PJ. Effect of delta-9tetrahydrocannabinol and cannabidiol on nocturnal sleep and earlymorning behavior in young adults. J Clin Psychopharmacol. (2004) 24:30513. doi: 10.1097/01.jcp.0000125688.05091.8f
33. Stephens RS, Babor TF, Kadden R, Miller M. The marijuana treatment project: rationale, design and participant characteristics. Addiction. (2002) 97:109-24. doi: 10.1046/j.1360-0443.97.s01.6.x

34. Halikas JA, Weller RA, Morse CL, Hoffmann RG. A longitudinal study of marijuana effects. Int $J$ Addict. (1985) 20:701-11. doi: 10.3109/10826088509044290

35. Ogeil RP, Phillips JG, Rajaratnam SMW, Broadbear JH. Risky drug use and effects on sleep quality and daytime sleepiness. Hum Psychopharmacol Clin Exp. (2015) 30:356-63. doi: 10.1002/hup.2483

36. Neylan TC, Mueller SG, Wang Z, Metzler TJ, Lenoci M, Truran D, et al. Insomnia severity is associated with a decreased volume of the CA3/dentate gyrus hippocampal subfield. Biol Psychiatry. (2010) 68:494-6. doi: 10.1016/j.biopsych.2010.04.035

37. Van Essen DC, Ugurbil K, Auerbach E, Barch DM, Behrens TE, Bucholz $\mathrm{R}$, et al. The human connectome project: a data acquisition perspective. Neuroimage. (2012) 62:2222-31. doi: 10.1016/j.neuroimage.2012.02.018

38. Herkenham M, Lynn AB, Litrle MD, Johnsont MR, Melvin LS, De Costa BR, et al. Cannabinoid receptor localization in brain. Proc Natl Acad Sci. (1990) 87:1932-6. doi: 10.1073/pnas.87.5.1932

39. Van Essen DC, Smith SM, Barch DM, Behrens TEJ, Yacoub E, Ugurbil K, et al. The WU-Minn human connectome project: an overview. NeuroImage. (2013) 80:62-79.

40. Manza P, Shokri-Kojori E, Volkow ND. Reduced Segregation Between Cognitive and Emotional Processes in Cannabis Dependence. Cereb Cortex. (2020) 30:628-39. doi: 10.1093/cercor/bhz113

41. Orr JM, Paschall CJ, Banich MT. Recreational marijuana use impacts white matter integrity and subcortical (but not cortical) morphometry. Neuroimage Clin. (2016) 12:47-56. doi: 10.1016/j.nicl.2016.06.006

42. Manza P, Tomasi DG, Volkow ND. Subcortical local functional hyperconnectivity in cannabis dependence. Biol Psychiatry Cogn Neurosci Neuroimaging. (2018) 3:285-93. doi: 10.1016/j.bpsc.2017.11.004

43. Glasser MF, Sotiropoulos SN, Anthony Wilson J, Coalson TS, Fischl B, Andersson JL, et al. The minimal preprocessing pipelines for the Human Connectome Project. Neuroimage. (2013) 80:105-124.

44. Desikan RS, Ségonne F, Fischl B, Quinn BT, Dickerson BC, Blacker D, et al. An automated labeling system for subdividing the human cerebral cortex on MRI scans into gyral based regions of interest. Neuroimage. (2006) 31:968-80. doi: 10.1016/j.neuroimage.2006.01.021

45. Buysse DJ, Reynolds CF, Monk TH, Berman SR, Kupfer DJ. The Pittsburgh sleep quality index: a new instrument for psychiatric practice and research. Psychiatry Res. (1989) 28:193-213. doi: 10.1016/0165-1781(89) 90047-4

46. Tingley D, Yamamoto $\mathrm{T}$, Hirose $\mathrm{K}$, Keele L, Imai K. Mediation: $\mathrm{R}$ package for causal mediation analysis. J Stat Softw. (2014) 59:1-38. doi: $10.18637 /$ jss.v059.i05

47. Owens MM, Sweet LH, MacKillop J. Recent cannabis use is associated with smaller hippocampus volume: high-resolution segmentation of structural subfields in a large non-clinical sample. Addict Biol. (2020) 26:e12874. doi: $10.1111 / \mathrm{adb} .12874$

48. Paul S, Bhattacharyya S. Cannabis use-related working memory deficit mediated by lower left hippocampal volume. Addict Biol. (2020). doi: 10.1111/adb.12984. [Epub ahead of print].

49. Cabeen RP, Allman JM, Toga AW. THC exposure is reflected in the microstructure of the cerebral cortex and amygdala of young adults. Cereb Cortex. (2020) 30:4949-63. doi: 10.1093/cercor/bhaa087

50. Marsden JF. Cerebellar ataxia. In: Day BL, Lord SR, editors. Handbook of Clinical Neurology. Plymouth: Elsevier B.V. p. 261-281.

51. Saab CY, Willis WD. The cerebellum: organization, functions and its role in nociception. Brain Res Rev. (2003) 42:85-95. doi: 10.1016/S0165-0173(03)00151-6

52. Fuentes CT, Bastian AJ. "Motor cognition" - what is it and is the cerebellum involved? Cerebellum. (2007) 6:232-6. doi: 10.1080/14734220701329268

53. Koziol LF, Budding D, Andreasen N, D’Arrigo S, Bulgheroni S, Imamizu H, et al. Consensus paper: the cerebellum's role in movement and cognition. Cerebellum. (2014) 13:151-77. doi: 10.1007/s12311-013-0511-x

54. Volkow ND, Gillespie H, Mullani N, Tancredi L, Grant C, Ivanovic $\mathrm{M}$, et al. Cerebellar metabolic activation by delta-9-tetrahydrocannabinol 
in human brain: a study with positron emission tomography and $18 \mathrm{~F}-$ 2-Fluoro-2-deoxyglucose. Psychiatry Res Neuroimaging. (1991) 40:69-78. doi: 10.1016/0925-4927(91)90030-T

55. Blithikioti C, Miquel L, Batalla A, Rubio B, Maffei G, Herreros I, et al. Cerebellar alterations in cannabis users: a systematic review. Addict Biol. (2019) 24:1121-37. doi: 10.1111/adb.12714

56. Sweigert J, Pagulayan K, Greco G, Blake M, Larimer M, Kleinhans NM. A multimodal investigation of cerebellar integrity associated with high-risk cannabis use. Addict Biol. (2020) 25:1-18. doi: 10.1111/adb. 12839

57. Wang Y, Zuo C, Xu Q, Hao L. Cerebellar thickness changes associated with heavy cannabis use: a 3-year longitudinal study. Addict Biol. (2020) 26:12-5. doi: 10.1111/adb.12931

58. Chindemi C, Cirielli V, Cima L, Danzi O, Raniero D, Tagliaro F, et al. Autophagy pathways in drug abusers after forensic autopsy: LC3B, ph-mTOR and p70S6K analysis. Med Sci Law. (2019) 59:49-56. doi: 10.1177/0025802419828910

59. Balandin AA, Zheleznov LM, Balandina IA, Balandin VA, Borodulin DV. Morfologicheskaya kharakteristika kory mozzhechka v molodom vozraste i izmeneniya ee tsitoarkhitektoniki pri opioidnoi zavisimosti [Morphological characteristics of the cerebellar cortex at a young age and changes in its cytoarchitectonics in opioid dependence]. Sud Med Ekspert. (2021) 64:18-22. doi: 10.17116/sudmed20216402118

60. Gil-Miravet I, Guarque-Chabrera J, Carbo-Gas M, Olucha-Bordonau F, Miquel M. The role of the cerebellum in drug-cue associative memory: functional interactions with the medial prefrontal cortex. Eur J Neurosci. (2019) 50:2613-22. doi: 10.1111/ejn.14187

61. Hung CC, Zhang S, Chen CM, Duann JR, Lin CP, Lee TS, et al. Striatal functional connectivity in chronic ketamine users: a pilot study. Am J Drug Alcohol Abuse. (2020) 46:31-43. doi: 10.1080/00952990.2019.1624764

62. Herreros I, Miquel L, Blithikioti C, Nuño L, Rubio Ballester B, Grechuta $\mathrm{K}$, et al. Motor adaptation impairment in chronic cannabis users assessed by a visuomotor rotation task. J Clin Med. (2019) 8:1049. doi: $10.3390 / \mathrm{jcm} 8071049$

63. Nurmedov S, Metin B, Ekmen S, Noyan O, Yilmaz O, Darcin A, et al. Thalamic and cerebellar gray matter volume reduction in synthetic cannabinoids users. Eur Addict Res. (2015) 21:315-20. doi: $10.1159 / 000430437$

64. Moulton EA, Elman I, Becerra LR, Goldstein RZ, Borsook D. The cerebellum and addiction: insights gained from neuroimaging research. Addict Biol. (2014) 19:317-31. doi: 10.1111/adb.12101

65. Miquel M, Vazquez-Sanroman D, Carbo-Gas M, Gil-Miravet I, SanchisSegura C, Carulli D, et al. Have we been ignoring the elephant in the room? Seven arguments for considering the cerebellum as part of addiction circuitry. Neurosci Biobehav Rev. (2016) 60:1-11. doi: 10.1016/j.neubiorev.2015.11.005

66. Moreno-Rius J, Miquel M. The cerebellum in drug craving. Drug Alcohol Depend. (2017) 173:151-8. doi: 10.1016/j.drugalcdep.2016.12.028

67. Kawamura Y. The CB1 cannabinoid receptor is the major cannabinoid receptor at excitatory presynaptic sites in the hippocampus and cerebellum. J Neurosci. (2006) 26:2991-3001. doi: 10.1523/JNEUROSCI.4872-0 5.2006

68. Iversen L. Cannabis and the brain. Brain. (2003) 126:1252-70. doi: 10.1093/brain/awg143

69. Hirvonen J, Goodwin RS, Li C-T, Terry GE, Zoghbi SS, Morse C, et al. Reversible and regionally selective downregulation of brain cannabinoid CB1 receptors in chronic daily cannabis smokers. Mol Psychiatry. (2012) 17:642-9. doi: 10.1038/mp.2011.82

70. Cousijn J, Wiers RW, Ridderinkhof KR, van den Brink W, Veltman DJ, Goudriaan AE. Grey matter alterations associated with cannabis use: results of a VBM study in heavy cannabis users and healthy controls. Neuroimage. (2012) 59:3845-51. doi: 10.1016/j.neuroimage.2011.09.046

71. Battistella G, Fornari E, Annoni J-M, Chtioui H, Dao K, Fabritius $\mathrm{M}$, et al. Long-Term effects of cannabis on brain structure. Neuropsychopharmacology. (2014) 39:2041-8. doi: 10.1038/npp.2014.67

72. Wu YF, Yang B. Gray matter changes in chronic heavy cannabis users: a voxel-level study using multivariate pattern analysis approach. Neuroreport. (2020) 31:1236-41. doi: 10.1097/WNR.0000000000001532
73. Koenders L, Cousijn J, Vingerhoets WA, van den Brink W, Wiers RW, Meijer CJ, et al. Grey matter changes associated with heavy cannabis use: a longitudinal sMRI study. PLoS ONE. (2016) 11:e0152482. doi: 10.1371/journal.pone.0152482

74. Becker JB, McClellan ML, Reed BG. Sex differences, gender and addiction. J Neurosci Res. (2017) 95:136-47. doi: 10.1002/jnr.23963

75. Greaves L, Hemsing N. Sex and gender interactions on the use and impact of recreational cannabis. Int J Environ Res Public Health. (2020) 17:509. doi: 10.3390/ijerph17020509

76. Yücel M, Solowij N, Respondek C, Whittle S, Fornito A, Pantelis C, et al. Regional brain abnormalities associated with long-term heavy cannabis use. Arch Gen Psychiatry. (2008) 65:694-701. doi: 10.1001/archpsyc.65. 6.694

77. McQueeny T, Padula CB, Price J, Medina KL, Logan P, Tapert SF. Gender effects on amygdala morphometry in adolescent marijuana users. Behav Brain Res. (2011) 224:128-34. doi: 10.1016/j.bbr.2011.05.031

78. Huang Z, Liang P, Jia X, Zhan S, Li N, Ding Y, et al. Abnormal amygdala connectivity in patients with primary insomnia: evidence from resting state fMRI. Eur J Radiol. (2012) 81:1288-95. doi: 10.1016/j.ejrad.2011.03.029

79. Klumpp H, Hosseini B, Phan KL. Self-reported sleep quality modulates amygdala resting-state functional connectivity in anxiety and depression. Front Psychiatry. (2018) 9:220. doi: 10.3389/fpsyt.2018.00220

80. Brabec J, Rulseh A, Horinek D, Pala A, Guerreiro H, Buskova J, et al. Volume of the amygdala is reduced in patients with narcolepsy - a structural MRI study. Neuro Endocrinol Lett. (2011) 32:652-6.

81. Neumeister A, Normandin MD, Pietrzak RH, Piomelli D, Zheng MQ, Gujarro-Anton A, et al. Elevated brain cannabinoid CB1 receptor availability in post-traumatic stress disorder: a positron emission tomography study. Mol Psychiatry. (2013) 18:1034-40. doi: 10.1038/mp.2013.61

82. Normandin MD, Zheng M-Q, Lin K-S, Mason NS, Lin S-F, Ropchan J, et al. Imaging the cannabinoid CB1 receptor in humans with $[11 \mathrm{C}]$ OMAR: assessment of kinetic analysis methods, test-retest reproducibility, and gender differences. J Cereb Blood Flow Metab. (2015) 35:1313-22. doi: 10.1038/jcbfm.2015.46

83. D'Souza DC, Cortes-Briones JA, Creatura G, Bluez G, Thurnauer H, Deaso E, et al. Efficacy and safety of a fatty acid amide hydrolase inhibitor (PF-04457845) in the treatment of cannabis withdrawal and dependence in men: a double-blind, placebo-controlled, parallel group, phase 2a single-site randomised controlled trial. Lancet Psychiatry. (2019) 6:35-45. doi: 10.1016/S2215-0366(18)30427-9

84. Schacht JP, Hutchison KE, Filbey FM. Associations between cannabinoid receptor-1 (CNR1) variation and hippocampus and amygdala volumes in heavy cannabis users. Neuropsychopharmacology. (2012) 37:2368-76. doi: 10.1038/npp.2012.92

85. Sonni A, Kurdziel LBF, Baran B, Spencer RMC. The effects of sleep dysfunction on cognition, affect, and quality of life in individuals with cerebellar ataxia. J Clin Sleep Med. (2014) 10:535-43. doi: 10.5664/jcsm.3706

86. Pedroso JL, Braga-Neto P, Felício AC, Aquino CCH, Prado LBF do, Prado GF do, et al. Sleep disorders in cerebellar ataxias. Arq Neuropsiquiatr. (2011) 69:253-7. doi: 10.1590/S0004-282X2011000200021

87. Winiger EA, Hitchcock LN, Bryan AD, Cinnamon Bidwell L. Cannabis use and sleep: expectations, outcomes, and the role of age. Addict Behav. (2021) 112:106642. doi: 10.1016/j.addbeh.2020.106642

88. Winiger EA, Huggett SB, Hatoum AS, Stallings MC, Hewitt JK. Onset of regular cannabis use and adult sleep duration: genetic variation and the implications of a predictive relationship. Drug Alcohol Depend. (2019) 204:107517. doi: 10.1016/j.drugalcdep.2019.06.019

89. Volkow ND, Swanson JM, Evins AE, DeLisi LE, Meier MH, Gonzalez $\mathrm{R}$, et al. Effects of cannabis use on human behavior, including cognition, motivation, and psychosis: a review. JAMA Psychiatry. (2016) 73:292-7. doi: 10.1001/jamapsychiatry.2015.3278

90. Glasser MF, Smith SM, Marcus DS, Andersson JLR, Auerbach EJ, Behrens TEJ, et al. The human connectome project's neuroimaging approach. Nat Neurosci. (2016) 19:1175-87. doi: 10.1038/nn.4361

91. Arterberry BJ, Treloar Padovano H, Foster KT, Zucker RA, Hicks BM. Higher average potency across the United States is associated with progression to first cannabis use disorder symptom. Drug Alcohol Depend. (2019) 195:18692. doi: 10.1016/j.drugalcdep.2018.11.012 
92. Hemsing N, Greaves L. Gender norms, roles and relations and cannabis-use patterns: a scoping review. Int J Environ Res Public Health. (2020) 17:947. doi: 10.3390/ijerph17030947

93. Cranford JA, Eisenberg D, Serras AM. Substance use behaviors, mental health problems, and use of mental health services in a probability sample of college students. Addict Behav. (2009) 34:134-45. doi: 10.1016/j.addbeh.2008.09.004

94. Carliner H, Mauro PM, Brown QL, Shmulewitz D, Rahim-Juwel R, Sarvet $\mathrm{AL}$, et al. The widening gender gap in marijuana use prevalence in the U.S. during a period of economic change, 2002-2014. Drug Alcohol Depend. (2017) 170:51-8. doi: 10.1016/j.drugalcdep.2016.10.042

95. Felton JW, Collado A, Shadur JM, Lejuez CW, MacPherson L. Sex differences in self-report and behavioral measures of disinhibition predicting marijuana use across adolescence. Exp Clin Psychopharmacol. (2015) 23:265-74. doi: $10.1037 /$ pha0000031

96. Farmer RF, Kosty DB, Seeley JR, Duncan SC, Lynskey MT, Rohde P, et al. Natural course of cannabis use disorders. Psychol Med. (2015) 45:63-72. doi: $10.1017 /$ S003329171400107X

97. Johnson RM, Fairman B, Gilreath T, Xuan Z, Rothman EF, Parnham $T$, et al. Past 15-year trends in adolescent marijuana use: differences by race/ethnicity and sex. Drug Alcohol Depend. (2015) 155:8-15. doi: 10.1016/j.drugalcdep.2015.08.025
98. Cuttler C, Mischley LK, Sexton M. Sex differences in cannabis use and effects: a cross-sectional survey of Cannabis users. Cannabis Cannabinoid Res. (2016) 1:166-75. doi: 10.1089/can.2016.0010

99. Kuhathasan N, Dufort A, MacKillop J, Gottschalk R, Minuzzi L, Frey BN. The use of cannabinoids for sleep: a critical review on clinical trials. Exp Clin Psychopharmacol. (2019) 27:383-401. doi: 10.1037/pha00 00285

100. Volkow ND, Weiss SRB. Importance of a standard unit dose for cannabis research. Addiction. (2020) 115:1219-21. doi: 10.1111/add. 14984

Conflict of Interest: The authors declare that the research was conducted in the absence of any commercial or financial relationships that could be construed as a potential conflict of interest.

Copyright $\odot 2021$ McPherson, Tomasi, Wang, Manza and Volkow. This is an openaccess article distributed under the terms of the Creative Commons Attribution License (CC BY). The use, distribution or reproduction in other forums is permitted, provided the original author(s) and the copyright owner(s) are credited and that the original publication in this journal is cited, in accordance with accepted academic practice. No use, distribution or reproduction is permitted which does not comply with these terms. 